\title{
Pc5 geomagnetic fluctuations in response to solar wind excitation and their relationship with relativistic electron fluxes in the outer radiation belt
}

\author{
Mauro Regi, Marcello De Lauretis and Patrizia Francia
}

\begin{abstract}
This study is focused to investigate the Pc5 geomagnetic pulsations in response to the solar wind forcing and their relationship with the relativistic electron flux at geostationary orbit. We analyzed the correlation of the Pc5 power in the magnetosphere and on the ground, at low and high latitude, with the solar wind speed and fluctuation power of the interplanetary magnetic field and solar wind dynamic pressure through the years 2006 to 2010, also examining the relative timing between pulsations and solar wind parameters. We found a very significant correlation of the Pc5 power with simultaneous solar wind pressure fluctuations and with the solar wind speed lagged by several hours; the relative amplitude of the two correlation peaks depending on the solar cycle phase and on the latitude.

We also found a strong relationship between the Pc5 power and the $>600 \mathrm{keV}$ and $>2 \mathrm{MeV}$ electron flux at geosynchronous orbit. Clear evidence emerges that the electron flux follows the Pc5 power by about 2 days; the time delay is a bit longer for the higher energy electrons.
\end{abstract}

Keywords: Solar wind; ULF waves; Magnetospheric relativistic electrons

\section{Background}

Ultra-low-frequency (ULF, $1 \mathrm{mHz}$ to $5 \mathrm{~Hz}$ ) fluctuations of the geomagnetic field have received in recent years considerable attention as being involved in the acceleration of magnetospheric electrons in the radiation belts (Menk 2011). On this regard, several studies have shown that intense and persistent activity of ULF waves in the low frequency range (1.7 to $6.7 \mathrm{mHz}$, Pc5 pulsations), observed at auroral latitudes, was followed within 1 to 2 days by enhanced fluxes of relativistic electrons (approximately $\mathrm{MeV}$ ) at geosynchronous orbit (Rostoker et al. 1998; Baker et al. 1998; Mathie and Mann 2001; Mann et al. 2004). Pc5 pulsations in the magnetosphere are traditionally believed to be driven by the KelvinHelmholtz instability (KHI) along the flanks of the magnetopause (Southwood 1968; Kivelson and Pu 1984); the compressional waves due to the $\mathrm{KHI}$ action, propagating earthward, can couple resonantly with the Alfven modes

\footnotetext{
* Correspondence: mauro.regi@aquila.infn.it

Department of Physical and Chemical Sciences, University of L'Aquila, 67100 L'Aquila, Italy
}

\section{勿

of the magnetospheric field lines (Southwood 1974; Chen and Hasegawa 1974). A statistical analysis has been recently focused on the resonant wave characteristics in the outer magnetosphere (Kokubun 2013). Such pulsations are observed also on the ground as a result of the ionospheric currents induced by the magnetospheric signals. Since the frequency of the Pc5 waves is comparable to the trapped electron drift frequency, acceleration can occur through resonant interaction of the ULF electric and magnetic field oscillations with the electron drift motion, leading to violation of the third adiabatic invariant and inward radial transport of electrons (Schulz and Lanzerotti 1974; Elkington 2006; Millan and Baker 2012).

The observed close correspondence between Pc5 intense activity and high values of the solar wind (SW) speed could indicate that the pulsation source is the KelvinHelmholtz instability at the magnetopause (Rostoker et al. 1998; Engebretson et al. 1998; Mann et al. 2004). Interestingly, however, the highest correlation was found with the SW speed lagged by approximately 1 day with respect to the Pc5 power, i.e., during the rising phase of fast SW 
speed streams (Engebretson et al. 1998; Mann et al. 2004); such result, which appears somewhat inconsistent with the KHI driving mechanism, has been explained by Engebretson et al. (1998) still in terms of the KHI, pointing out that, in addition to the SW speed, the compression at the leading edge of fast SW streams could increase the growth rate of the instability. On the other hand, since the magnetopause responds directly to changes of the SW dynamic pressure (Sarafopoulos 1995; Francia et al. 1999), SW pressure fluctuations could be directly transmitted in the magnetosphere via magnetopause buffeting (Kepko et al. 2002; Mann et al. 2004). Kessel (2008) examined the relationship between Pc5 wave power in the SW, near the magnetopause, in the magnetosphere at geostationary orbit and over the poles and on the ground during high-speed streams and coronal mass ejections; over $80 \%$ of total Pc5 activity during a 2-week interval was found to be driven by SW pressure fluctuations. Moreover, Takahashi and Ukhorskiy (2008), investigating through a cross-correlation analysis the relationship between SW parameters and magnetospheric Pc5 waves at geosynchronous orbit during 2006, observed that the SW pressure fluctuation power had a higher correlation with the magnetospheric Pc5 power than the SW speed at or very near to a zero time shift. They concluded that the major driver of geosynchronous Pc5 pulsations are the SW pressure variations instead of the KHI and, on the other hand, explained the time shift of approximately -1 day of the maximum correlation between the SW speed and magnetospheric Pc5 power (the Pc5 power preceding the speed) in terms of the intrinsic time delay between the SW pressure fluctuation power and speed. A more recent statistical analysis (Takahashi et al. 2012), conducted using ground data at different latitudes during 2001 and 2006, showed that the pulsation amplitude dependence on SW speed (dynamic pressure fluctuation amplitude) was stronger at high (low) latitude, with the relative importance of the two SW parameters switching at $\mathrm{L} \sim 5$; the authors attributed the speed correlation to KHI (more efficient at high latitudes) and the pressure fluctuation correlation to global compression of the magnetosphere. Berube et al. (2014) found similar results using magnetospheric data by THEMIS during 2007 to 2009; the greater Pc5 power enhancement was associated to the SW speed in the outer magnetosphere beyond $\mathrm{L} \sim 6$ and to the pressure fluctuations at distances farther from the magnetopause and closer to Earth.

We found it would be interesting to further investigate the dependence of the ULF wave activity, observed both at geosynchronous orbit and on the ground, on the SW parameters and its relationship with the relativistic electron flux in the magnetosphere. To this purpose, in the present study, we analyzed the correlation of the Pc5 power with the SW speed, the magnetic and dynamic pressure fluctuation power, and the geosynchronous electron flux, also examining the relative timing, through the years 2006 to 2010, which correspond to the declining phase of the solar cycle 23 and to the onset of the solar cycle 24.

Our results show clearly that the Pc5 power is highly correlated with simultaneous SW pressure fluctuations and with the SW speed lagged by several hours, the relative amplitude of the two correlation peaks depending on the solar cycle phase and, for ground pulsations, on the latitude.

The correlation of the Pc5 power with the relativistic electron flux is high, with the electron flux delayed by approximately 2 days.

\section{Methods}

The interplanetary medium conditions were monitored by using 1-min SW and interplanetary magnetic field (IMF) data, time-shifted to the Earth's bow shock nose, from OMNIweb (http://cdaweb.gsfc.nasa.gov). The 1min magnetospheric field data were from the fluxgate magnetometer on GOES 12 (Singer et al. 1996) while the relativistic electron flux data at 5-min time resolution were from the energetic particle sensor (EPS) on GOES 10 (2006) and GOES 11 (2007 to 2010) (Onsager et al. 1996). All GOES data were obtained from CDAWeb. On the ground, we analyzed the geomagnetic field fluctuations measured at L'Aquila (AQU) in Italy, and at Terra Nova Bay (TNB) in Antarctica, by triaxial searchcoil magnetometers sampled at $1 \mathrm{~s}$. The coordinates of the stations are shown in Table 1. While the AQU local field line lies in the inner magnetosphere, TNB is at the footprint of an open field line at the magnetosphere/SW boundary and approaches the cusp at local noon.

To analyze the fluctuations, we used the Fourier transform of 1-h time series (frequency resolution approximately $0.28 \mathrm{mHz}$ ); each spectrum was smoothed over nine frequency bands using a triangular moving window (final frequency resolution approximately $1.39 \mathrm{mHz}$ ). Magnetospheric and ground data were differenced before computing the spectra in order to reduce the original spectral slope and to perform the smoothing procedure on almost equalized data; the resulting spectra were then re-corrected by means of the transfer function of the difference filter. At ground, where the data are measured by search-coil magnetometers, the spectra are also converted, by means of the instrument transfer function, from $\mathrm{mV}^{2} / \mathrm{Hz}$ into $\mathrm{nT}^{2} / \mathrm{Hz}$.

We computed the Pc5 integrated spectral power of the IMF strength $P_{\mathrm{B}}, \mathrm{SW}$ dynamic pressure $P_{\mathrm{nV}}{ }^{2}$ magnetospheric field $P_{\mathrm{G} 12}$ (as obtained from the sum of the power on the three components $\left.P=P_{\mathrm{X}}+P_{\mathrm{Y}}+P_{\mathrm{Z}}\right)$, and ground field $P_{\mathrm{TNB}}$ and $P_{\mathrm{AQU}}$ (as obtained from the sum 
Table 1 Geographic and geomagnetic coordinates, MLT, and LT for ground stations and geosynchronous satellites

\begin{tabular}{|c|c|c|c|c|}
\hline Code name & Geographic coordinate & Corrected geomagnetic coordinates (IGRF05) & Magnetic local time (MLT) & Local time (LT) \\
\hline TNB & $74.69^{\circ} \mathrm{S}, 164.12^{\circ} \mathrm{E}$ & $80.01^{\circ} \mathrm{S}, 306.94^{\circ} \mathrm{E}$ & $\mathrm{MLT} \sim \mathrm{UT}-8$ & $\mathrm{LT} \sim \mathrm{UT}+11$ \\
\hline AQU & $42.38^{\circ} \mathrm{N}, 13.32^{\circ} \mathrm{E}$ & $36.33^{\circ} \mathrm{N}, 87.37^{\circ} \mathrm{E}$ & $\mathrm{MLT} \sim \mathrm{UT}+1$ & $\mathrm{LT} \sim \mathrm{UT}+1$ \\
\hline GOES 12 & - & - & $\mathrm{MLT} \sim \mathrm{UT}+19$ & $\mathrm{LT} \sim \mathrm{UT}+19$ \\
\hline GOES 10 and GOES 11 & - & - & $\mathrm{MLT} \sim \mathrm{UT}+15$ & $\mathrm{LT} \sim \mathrm{UT}+15$ \\
\hline
\end{tabular}

of the power on the $\mathrm{H}$ (northward) and $\mathrm{D}$ (eastward) horizontal components $\left.P=P_{\mathrm{H}}+P_{\mathrm{D}}\right)$. In order to remove the daily modulation from the TNB, AQU, and GOES power, we computed 24-h moving averages, with a step size of $3 \mathrm{~h}$; in addition, since the power $P$ typically undergoes changes of several orders of magnitude between adjacent measurements, in the average procedure we used $\log P$ that has a quasi-normal distribution instead of $P$. For comparison, also the SW parameters were averaged by the same procedure.

The geomagnetic field response to the interplanetary medium changes was analyzed through a cross-correlation analysis. For each 10-day time interval, the crosscorrelation coefficient $r_{\mathrm{i}}(x, y)$ was computed as a function of the lag between the input $x$ and output $y$ time series (converted to zero mean and unitary variance, in order to directly compare the fluctuations). Then, we computed the average cross-correlation coefficient $r(x, y)$ over all time intervals; a negative lag, associated at $r(x, y)$, indicates that $x$ precedes $y$. The corresponding $95 \%$ confidence interval was estimated through a Monte Carlo test on 10,000 random time series, computed on the basis of the actual degrees of freedom.

\section{Results and discussion}

In this section, we present the results of our correlation analysis conducted through the interval 2006 to 2010. In particular, in the section 'Magnetic field power at geosynchronous orbit and on the ground', we evaluated the correspondence between Pc5 magnetic field fluctuations at geosynchronous orbit and on the ground; in the section 'Interplanetary parameters and geomagnetic field fluctuations', we studied the correlation between SW parameters and geomagnetic field fluctuations, showing that the Pc5 geomagnetic power is essentially stimulated by the SW dynamic pressure fluctuations; in the section 'Energetic electron flux driven by geomagnetic pulsations', we show the correlation between the relativistic electron flux, recorded at geosynchronous orbit and the Pc5 power.

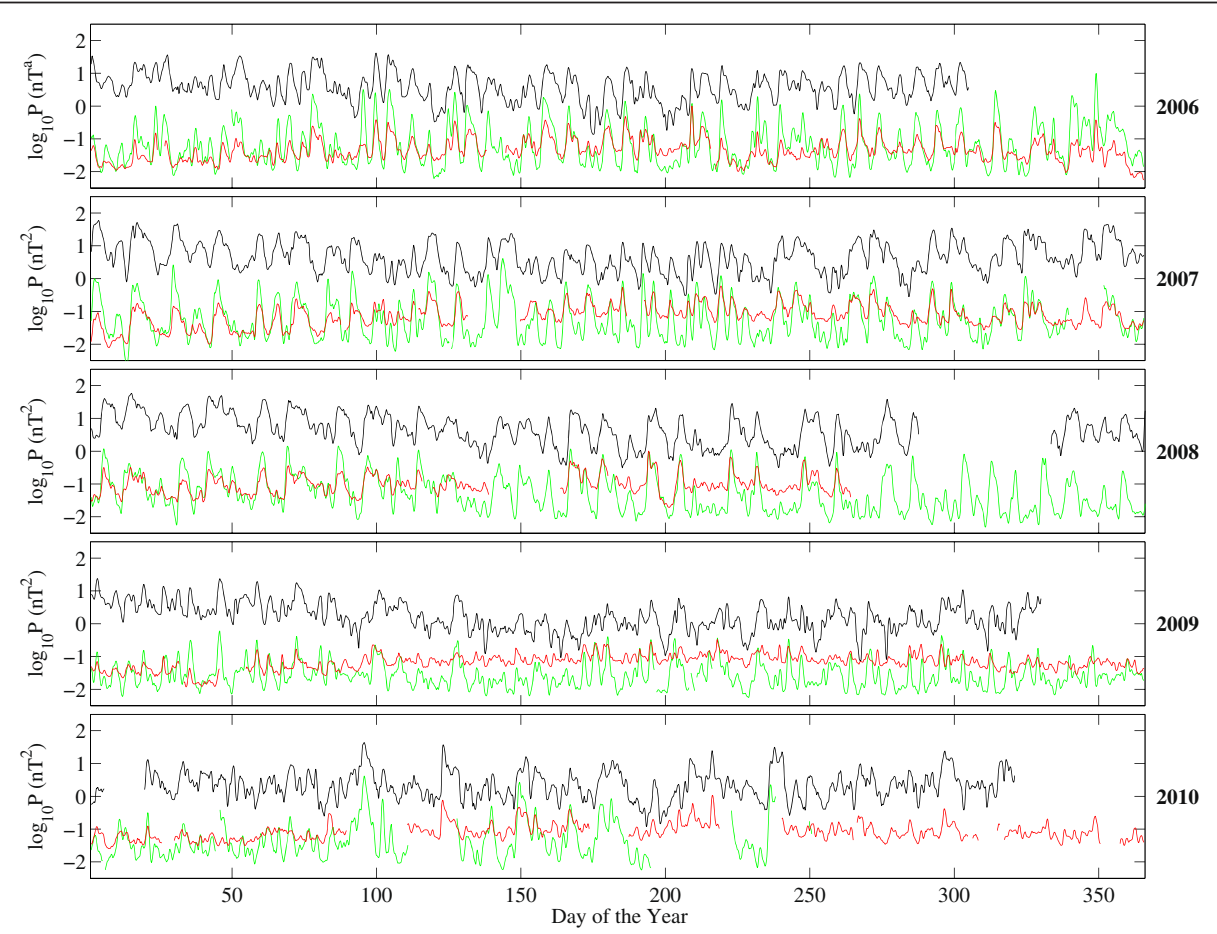

Figure 1 The magnetospheric and geomagnetic field fluctuation power. The Pc5 power recorded at TNB (black), AQU (red), and GOES 12 (green) for the years 2006 to 2010. 


\section{Magnetic field power at geosynchronous orbit and on the ground}

Figure 1 shows the time series of $\log P$ on the ground, at $\mathrm{AQU}$ and TNB, and at geosynchronous orbit through the years 2006 to 2010. During 2006 to 2008 and 2010, the different time series are very similar, showing a wellpronounced peak-to-peak correspondence; the correspondence tends to diminish during 2009 and at the beginning of 2010, when the power fluctuations are less regular, and their amplitude is smaller. The power shows larger relative fluctuations at geosynchronous orbit than on the ground (especially with respect to AQU), but in average, it is much higher at TNB, which is more affected by the magnetosphere/SW boundary processes.

The overall average cross-correlation coefficients between $\log P$ at geosynchronous orbit and on the ground are shown in Figure 2a. The maximum value of correlation is approximately 0.77 at TNB and approximately 0.73 at AQU, well above the $95 \%$ confidence level, and is found at a time lag of approximately $0 \mathrm{~h}$ at AQU and between 0 and $-3 \mathrm{~h}$ at TNB, suggesting that the fluctuations are almost simultaneously observed at AQU and GOES while they appear slightly delayed at TNB. Figure 2b shows the dynamic correlation analysis between $\log P$ at GOES and simultaneous $\log P$ at AQU and TNB; for a comparison, it also shows the SW speed $V_{\mathrm{sw}}$. The correlation is always significant (the $95 \%$ confidence levels estimated through the null hypothesis are about \pm 0.2 ) for both TNB and AQU (see, for example, Figure 2c), but it generally drops towards lower values in correspondence to low SW speed, and it is in average attenuated at the solar minimum, in absence of fast streams.

\section{Interplanetary parameters and geomagnetic field fluctuations}

The cross-correlation analysis between interplanetary parameters and magnetospheric and ground pulsations is shown in Figure 3 for each year. We compared the input parameters $V_{\mathrm{sw}}, \log P_{\mathrm{nV}}{ }^{2}$, and $\log P_{\mathrm{B}}$ with the output parameter $\log P_{\mathrm{G} 12}, \log P_{\mathrm{TNB}}$, and $\log P_{\mathrm{AQU}}$. For all the

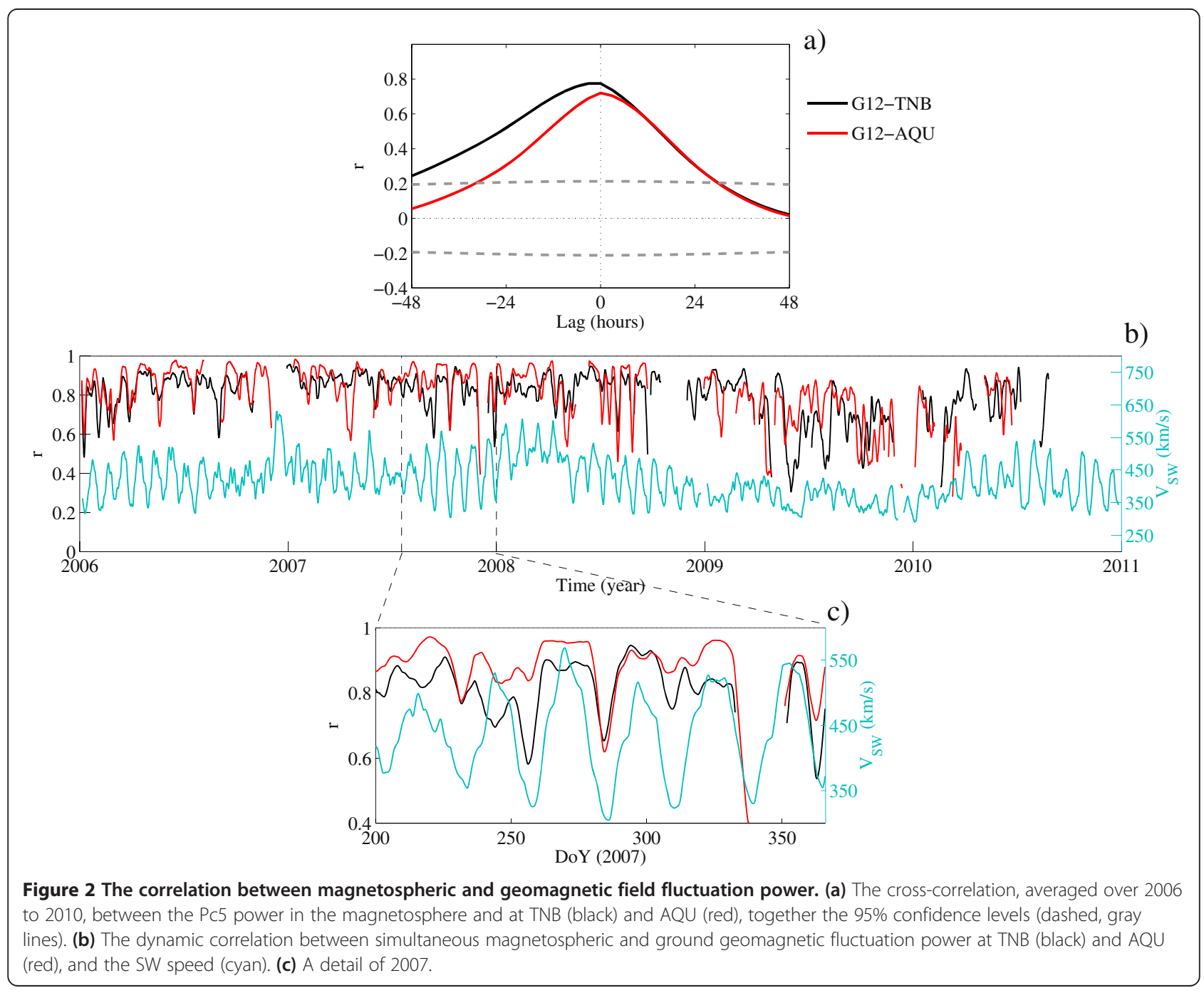




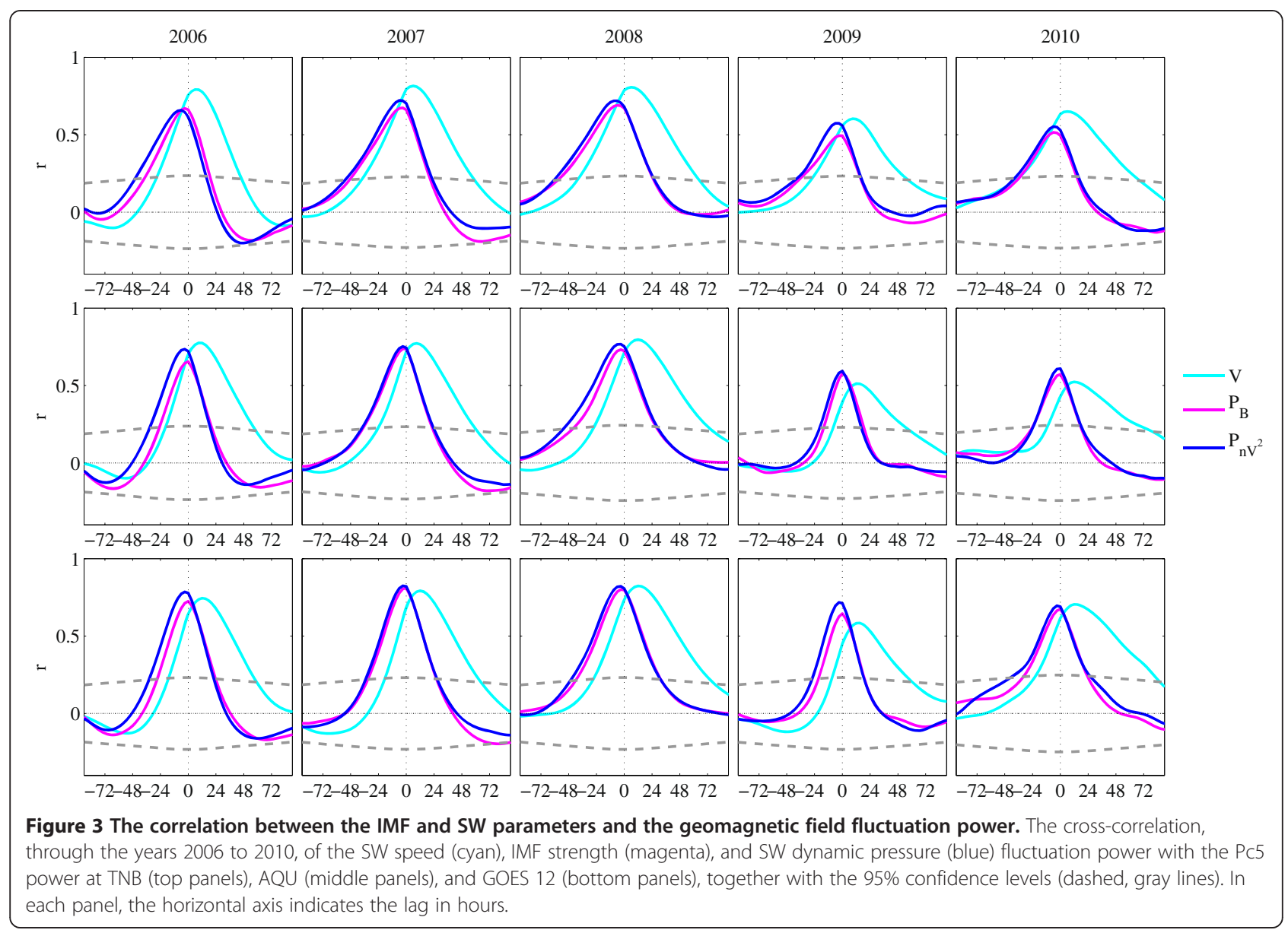

years, there is a strong correlation between the geosynchronous and ground Pc5 power and the fluctuation power in the IMF and SW pressure; the correlation peak is at a time lag ranging from 0 to $-3 \mathrm{~h}$, indicating that the geomagnetic pulsations follow with a slight delay the IMF and SW pressure fluctuations. In addition, a clear correlation with $V$ sw is observed with the geomagnetic pulsation power preceding the SW speed by a few hours; the time delay is shorter at TNB ( 6 to $9 \mathrm{~h})$ than at GOES and AQU (9 to $12 \mathrm{~h}$ ).

In all cases, as observed in Figure 2, the correlation reduces with the decreasing solar activity, reaching lower values during 2009 to 2010. At GOES, the pulsation power is better correlated with the SW compressional fluctuations than with $V_{\mathrm{sw}}$, while at TNB, we observe the opposite. At AQU, the correlation with the SW pressure fluctuations is approximately the same as with the SW speed during 2006 to 2008 and definitely higher close to the solar minimum. These features are in agreement with the results by Takahashi et al. (2012).

We remark that the geomagnetic pulsations correlate to the IMF and SW pressure fluctuations at a near zero time shift, indicating an essentially instantaneous driving at GOES and ground stations. The correlation with the SW speed delayed by few hours could be explained assuming that such time delay corresponds to the time interval separating the IMF and SW pressure fluctuations in the interaction region between slow and fast SW, i.e., upstream of the SW speed increase, from the high-speed stream (Takahashi and Ukhorskiy 2008). The higher correlation with $V_{\mathrm{sw}}$ at TNB and the associated shorter time delay probably indicate that on the outermost geomagnetic field lines the $\mathrm{KHI}$, growing with the SW speed increase, provides energy to fluctuations to persist during the high-speed conditions, slightly longer than at AQU or in the inner magnetosphere.

In Figure 4, we show, as an example, the SW and geomagnetic time series during March to April 2006. It clearly emerges that the $V_{\mathrm{sw}}$ maximum (marked by vertical dashed lines) follows the Pc5 power maximum of the magnetospheric, ground, SW pressure, and IMF fluctuations, mostly at time delays of several hours, consistently with the values observed in Figure 3. 


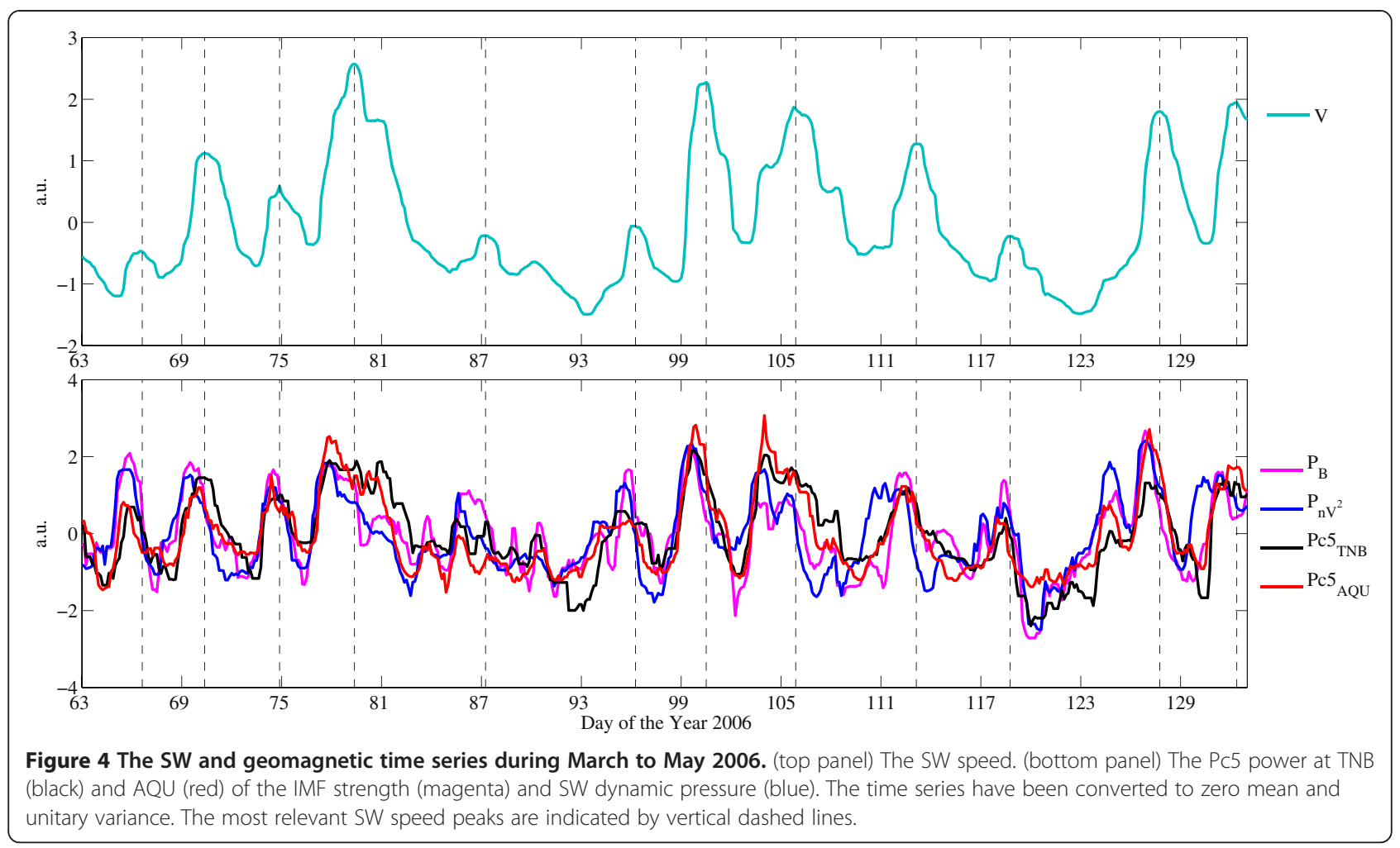

Furthermore, such time delays can be considered consistent with the approximately 1-day delay reported by Mann et al. (2004), using data with 1-day resolution. Engebretson et al. (1998), who found that high-latitude Pc5 power was stronger at the leading edge of SW streams, focused the attention on the KHI process and, using simulated SW speed and density and a constant IMF, computed a KHI parameter from the instability condition, to demonstrate that the magnetopause instability is expected after the sharp increase in density, at the leading edge of the high-speed stream, and is largest before the peak of the SW velocity. We found it interesting to compute the KHI parameter following their procedure. As an example, we show in Figure 5 the KHI parameter evaluated during two time intervals, respectively corresponding to days 125 to 129 of the year 2006 and days 200 to 204 of the year 2007, when SW streams reached the Earth's magnetosphere. In the figure, we show the SW data and the KHI parameter, together with the $\log P_{\mathrm{nV}}{ }^{2}, \log P_{\mathrm{B}}, \log P_{\mathrm{TNB}}$, and $\log P_{\mathrm{AQU}}$ time series. In both the events, it can be seen that $\log P_{\mathrm{nV}}{ }^{2}$ and $\log P_{\mathrm{B}}$ reach the highest values in correspondence to the compression region, before the SW speed steep increase, and are immediately followed by the $\log P_{\mathrm{TNB}}$ and $\log P_{\mathrm{AQU}}$ enhancements. On the other hand, the largest values of the KHI parameter occur when $V_{\mathrm{sw}}$ is still rising and follow the peak of $\log P_{\mathrm{TNB}}$ and $\log P_{\mathrm{AQU}}$ with a time delay of few hours. It is worth to note that the pulsation power at TNB persists high, longer than at AQU and in the SW and IMF fluctuations; this feature corresponds to persisting high values of the KHI parameter.

Our results show that the KHI is not the primary cause of the geomagnetic ULF activity in the Pc5 band, which rather seems to be due to the direct transfer of compressional waves into the magnetosphere; however, at TNB, the KHI seems to amplify the fluctuations during the whole high-SW speed period.

\section{Energetic electron flux driven by geomagnetic pulsations}

Finally, we compared the energetic electron flux at GOES 10 and 11 satellites and the geomagnetic pulsation power through the years. In Figure 6 (upper panel), we show the $>600 \mathrm{keV}$ electron flux and the Pc5 power at TNB (which, as seen in the section 'Magnetic field power at geosynchronous orbit and on the ground', was found very similar at AQU and GOES 12).Recurrent, almost simultaneous enhancements of the electron flux and Pc5 power characterize the years 2006 to 2008 and 2010, in correspondence to the occurrence of SW streams (shown in Figure 2); they tend to disappear during 2009, at the solar cycle minimum, when the average values of the flux and power strongly decrease, often becoming negligible. 


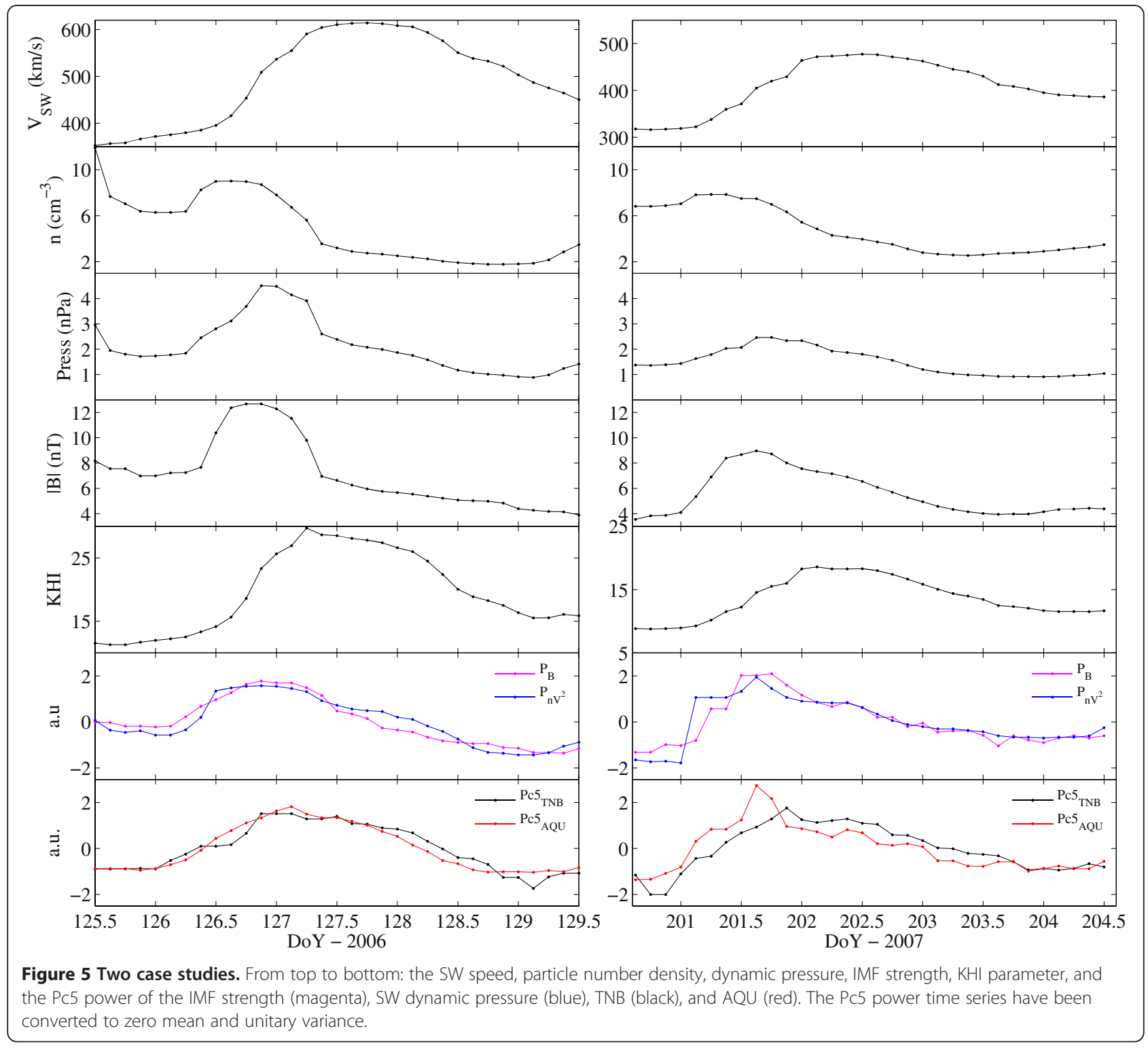

In the bottom panels of Figure 6, we show the results of the cross-correlation analysis between the geosynchronous $>600 \mathrm{keV}$ and $>2 \mathrm{MeV}$ electron flux and the Pc5 fluctuation power at AQU, TNB, and GOES 12; the analysis was restricted to the interval 2007 to 2008, during which the electron flux data coverage is almost continuous and the values are significantly high. The overall result is that, in both the energy ranges, the electron flux is well correlated with the Pc5 power at the three sites, more strongly at TNB, with the maximum correlation at a time delay of approximately 2 days. In more detail, the results show also a dependence on the energy; in particular, the electrons with energy $>600 \mathrm{keV}$ are more correlated and at a shorter time delay (1.8 to 2 days) with respect to the $>2 \mathrm{MeV}$ electrons (2 to 2.4 days), with an approximately 9 -h difference.

\section{Conclusions}

We investigated the dependence of geomagnetic Pc5 pulsations on SW speed and pressure fluctuations through the years 2006 to 2010 and analyzed their relationship with the magnetospheric energetic electron flux enhancements.

First, we found that the Pc5 pulsation power at geosynchronous orbit is closely correlated, at a zero lag, to the Pc5 power on the ground, although TNB pulsations tend to be slightly delayed (by 0 to $3 \mathrm{~h}$ ) with respect to GOES (and AQU). The correlation decreases during 

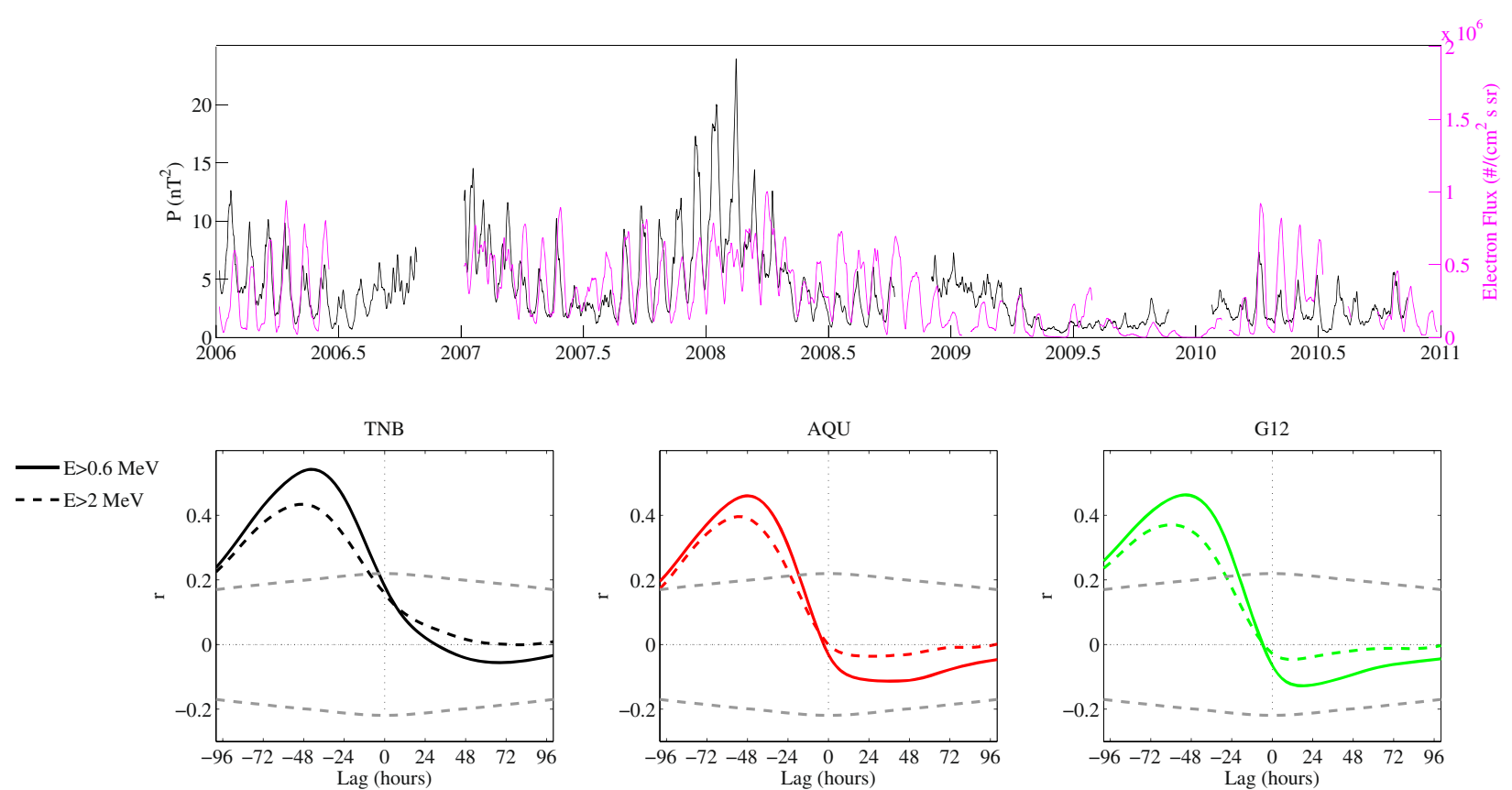

Figure 6 The relativistic electron flux and the Pc5 power. (top) The relativistic electron flux ( $>600 \mathrm{keV}$ ) at geosynchronous orbit (magenta) and the geomagnetic power at TNB (black). (bottom) The cross-correlation of the E $>600 \mathrm{keV}$ (solid) and E > $2 \mathrm{MeV}$ (dashed) electron flux with the PC5 power at TNB (black), AQU (red), and GOES 12 (green) during 2007 to 2008, together with the 95\% confidence levels (dashed, gray lines).

2009, when high-speed SW streams are rare and, as a consequence, the magnetospheric and ground signals driven by the SW, i.e., by a common source, are not so frequent as during the descending phase of the solar cycle.

A strong correlation was observed between the IMF and SW pressure fluctuation power and the Pc5 pulsation power, slightly time delayed. Such result indicates an almost instantaneous response of the magnetosphere to the IMF and SW pressure fluctuations, confirming their primary role in generating Pc5 pulsations. On the other hand, the pulsations are also related to the SW speed, preceding it by a few hours; such additional correlation is probably intrinsic due to the SW structure itself (Takahashi and Ukhorskiy 2008), since a fast SW stream typically follows by a few hours the interaction region where the IMF and SW pressure variations are largest. Some difference is observed, however, among GOES 12/AQU and TNB observations. While at geosynchronous orbit and at low latitude, the correlation with the IMF and SW pressure fluctuations is predominant; TNB pulsations are more correlated to the SW speed, preceding it by a shorter time interval with respect to AQU and GOES. These features indicate that, with respect to the inner magnetosphere, the KHI is important on the geomagnetic field line at the boundary of the magnetosphere, where it keeps high the pulsation power level as long as the SW speed remains high.
We also found a clear correlation of the energetic electron flux at geosynchronous orbit with the Pc5 power in the magnetosphere and at high and low latitude, at a time delay of approximately 2 days, in agreement with previous studies at auroral and middle latitudes (Rostoker et al. 1998; Baker et al. 1998; Mathie and Mann 2001; Mann et al. 2004). Such results are also consistent with those by Kozyreva et al. (2007) who correlated a ground, global ULF index with the $>2 \mathrm{MeV}$ electron flux at GOES 8 . The lower correlation peak and longer time delay observed for higher energy electrons confirm the results by Rodger et al. (2010), who found an energy-dependent time delay in the GOES 12 observations, with the $>2 \mathrm{MeV}$ electron enhancement somewhat time-delayed relative to the $>600 \mathrm{keV}$ electron enhancement, probably due to the timescales of the acceleration processes.

\section{Competing interests}

The authors declare that they have no competing interests.

\section{Authors' contributions}

All authors participated in the design of the study and data analysis. All authors read and approved the final manuscript.

\section{Acknowledgements}

This research activity was supported by the Italian PNRA (Programma Nazionale di Ricerche in Antartide). The authors acknowledge J.H. King and N. Papatashvilli at NASA and CDAWeb for solar wind data and $\mathrm{H}$. Singer and T. Onsager at NOAA SEC and CDAWeb for GOES satellites data of magnetic field and relativistic electron flux measurements. 
Received: 10 September 2014 Accepted: 4 January 2015

Published online: 22 January 2015

\section{References}

Baker DN, Pulkkinen TI, Li KX, Kanekal SG (1998) Coronal mass ejections, magnetic clouds and relativistic magnetospheric electron events: ISTP. J Geophys Res 103:17279-17291

Berube D, Sanny J, Taus R, Garoutte A (2014) Dayside distribution of Pc5 wave power in the quiet magnetosphere and its response to the solar wind. Planet Space Sci 97:1-8, doi:10.1016/j.pss.2014.04.012

Chen L, Hasegawa A (1974) A theory of long period magnetic pulsations, 1, steady state excitation of field line resonance. J Geophys Res 79:1024-1037

Elkington SR (2006) A review of ULF interactions with radiation belt electrons. Solar Eruptions and Energetic Particles Geophysical Monograph Series 165, AGU, doi:10.1029/169GM12, 177-193.

Engebretson M, Glassmeier K-H, Stellmacher M, Hughes WJ, Lühr H (1998) The dependence of high-latitude PC5 wave power on solar wind velocity and on the phase of high-speed solar wind streams. J Geophys Res 103(11):26,271-26,283

Francia P, Lepidi S, Villante U, Di Giuseppe P, Lazarus AJ (1999) Geomagnetic response at low latitude to continuous solar wind pressure variations during northward interplanetary magnetic field. J Geophys Res 104:19,923-19,930

Kepko L, Spence HE, Singer HJ (2002) ULF in the solar wind as direct drivers of magnetospheric pulsations. Geophys Res Lett 29:8, doi:10.1029/2001 GL014405

Kessel RL (2008) Solar wind excitation of PC5 fluctuations in the magnetosphere and on the ground. J Geophys Res 113:A04202, doi:10.1029/2007JA012255

Kivelson MG, Pu ZY (1984) The Kelvin-Helmholtz instability on the magnetopause. Planet Space Sci 32:1335-1341

Kokubun S (2013) ULF waves in the outer magnetosphere: geotail observation 1 transverse waves. Earth Planets Space 65:411-433

Kozyreva O, Pilipenko V, Engebretson MJ, Yumoto K, Watermann J, Romanova N (2007) In search of a new ULF wave index: comparison of PC5 power with dynamics of geostationary relativistic electrons. Planet Space Sci 55:755-769, doi:10.1016/j.pss.2006.03.013

Mann IR, O'Brien TP, Milling DK (2004) Correlations between ULF wave power, solar wind speed, and relativistic electron flux in the magnetosphere: solar cycle dependence. J Atmos Sol Terr Phy 66:187-198, doi:10.1016/j.jastp. 2003.10.002

Mathie RA, Mann IR (2001) On the solar wind control of PC5 ULF pulsation power at midlatitudes: implications for MeV electron acceleration in the outer radiation belt. J Geophys Res 106(12):9,783-29,796

Menk FW (2011) Magnetospheric ULF waves: a review. In: Liu W, Fujimoto M (eds) The dynamic magnetosphere, vol 3, AGA Special Sopron Book Series., pp 223-256

Millan RM, Baker DN (2012) Acceleration of particles to high energies in Earth's radiation belts. Space Sci Rev 173:103-131, doi:10.1007/s11214-012-9941-x

Onsager TG, Grubb R, Kunches J, Matheson L, Speich D, Zwickl R, Sauer H (1996) Operational uses of the GOES energetic particle detectors. Proc SPIE Int Soc Opt Eng 2812:281, in GOES-8 and Beyond: 7 - 9 August 1996. Denver, Colorado, edited by E. R. Washwell

Rodger CJ, Clilverd MA, Green JC, Lam MM (2010) Use of POES SEM-2 observations to examine radiation belt dynamics and energetic electron precipitation into the atmosphere. J Geophys Res 115:A04202, doi:10.1029/ 2008JA014023

Rostoker G, Skone S, Baker DN (1998) On the origin of relativistic electrons in the magnetosphere associated with some geomagnetic storms. Geophys Res Lett 25(19):3701-3704

Sarafopoulos DV (1995) Long duration Pc5 compressional pulsations inside the Earth's magnetotail lobes. Ann Geophys 13:926-937, doi:10.1007/s00585-9950926-x

Schulz M, Lanzerotti $L$ (1974) Particle diffusion in the radiation belts, vol 7. Phys. Chem. Space. Springer, New York, p 215

Singer HJ, Matheson L, Grubb R, Newman A, Bouwer SD (1996) Monitoring space weather with the GOES magnetometers, GOES-8 and beyond. Proc SPIE Int Soc Opt Eng 2812:299-308

Southwood DJ (1968) The hydromagnetic instability of the magnetospheric boundary. Planet Space Sci 16:587

Southwood DJ (1974) Some features of field line resonance in the magnetosphere. Planet Space Sci 22:482-491
Takahashi K, Ukhorskiy AY (2008) Timing analysis of the relationship between solar wind parameters and geosynchronous PC5 amplitude. J Geophys Res 113:A12204, doi:10.1029/2008JA013327

Takahashi K, Yumoto K, Claudepierre SG, Sanchez ER, Troshichev OA, Janzhura AS (2012) Dependence of the amplitude of Pc5-band magnetic field variations on the solar wind and solar activity. J Geophys Res 117:A04207, doi:10.1029/ 2011JA017120

\section{Submit your manuscript to a SpringerOpen ${ }^{\circ}$ journal and benefit from:}

- Convenient online submission

Rigorous peer review

- Immediate publication on acceptance

- Open access: articles freely available online

- High visibility within the field

- Retaining the copyright to your article

Submit your next manuscript at $>$ springeropen.com 